\title{
A Case for Identity Hierarchies in Simulating Social Groups
}

\author{
Platt A. ${ }^{1}$, Kneidl A. ${ }^{2}$ \\ 1,2 accu:rate $\mathrm{GmbH}$ \\ Rosental 5, Munich, Germany \\ ak@accu-rate.de; ap@accu-rate.de
}

\begin{abstract}
By considering previous empirical studies in group dynamics, modelling designs for pedestrian simulators and psychological and sociological theories of crowd behaviour, we briefly present a hierarchical, identity-based approach to simulating pedestrian social groups.
\end{abstract}

Keywords: Group modelling; Social identity; Simulation; Pedestrian modelling; Hierarchical structure

Social and emergent group behaviour in crowds has garnered enough attention that [1] present a review of research. The review provides a consolidation of and comment on empirical studies, most notably the prevalence of groups within crowds and some consistent phenomena: the ' $V$-shape' and the lower walking speed of groups compared to individuals (see e.g. [2], [3], [4] for further studies).

As with any simulation practice however, there is a struggle between empirical intricacy (in a universe of infinite parameters) and programmatic simplicity. There is strong evidence that heuristics robustly map cognition to action ${ }^{1}$, see [5] who suggest cognitive heuristics do not seek optimality but sufficiency through the use of an "adaptive [heuristic] toolbox". These ideas have been proposed and discussed extensively by [6] (also [11]). They hierarchically structure their heuristics, based on the concept of least-effort (e.g. [7, 8]). That is, heuristics are attempted successively; the heuristic of least effort is attempted first, then the second, until some goal is realized or postponed. In this way, environmental stimuli cause implicit changes in agent behaviour as a direct result of 'cognitive' functions. This approach lends itself to tackling emergent lane formation (e.g. [10]) and other phenomena where environmental conditions alter behavioural motives (as appose to environmental conditions directly acting on agents).

Exploiting and deriving heuristics, and even hierarchical heuristic toolboxes, is paramount to developing a meaningful group model. The questions for practitioners are: "what kind of information is used by the pedestrian?" and "how is this information processed to adapt the walking behavior?" [11].

The Social Identity Model of Deindividuation Effects (SIDE) may provide assistance. SIDE presents an agent with one or more social identities and a personal identity, each of which influences to a greater or lesser extent the behaviour of the agent [13]. SIDE traces its routes to observations in emergent, antisocial behaviour in crowds and mass gatherings (e.g. [14] $)^{2}$, however the conceptual distinction between social and personal identities seems both theoretically plausible (given its allegiance to the wellestablished social categorization theory [15]) and pragmatically useful. [16] already consider such identities to model helping behaviour amongst strangers during emergencies. However, we propose that it is SIDE's emphasis on the salience of social and personal identities, and the resultant partialdeindividuation (employed here as shared behavioural approaches) that should also be utilized in social group models.

As noted in the literature (e.g. [2], [3], [4]), group behaviour is dependent on both intrinsic properties of an agent: sex, age and mobility, and extrinsic properties of the group's relationship: family vs. friends, male friends vs. female friends etc. By considering an agent's personal and social identities, and hierarchically structuring these identities, such factors may be more easily confronted.

\footnotetext{
1 In fact, [12] suggests analogies are used before-the-fact; we anticipate how things might be based on previous, analogous "patterns and statistical regularities", before marrying our anticipations to reality. Use of such analogies and "context frames" could be considered, in an admittedly loose sense, heuristic-based perception (as an extension to heuristic-based action).

2 This is pertinent given [17]'s research report; evidently caution should be taken in determining social (identity) norms.
} 
In introducing personal and social identities to agent-based modelling, two main questions arise. First: under what circumstances do these identities manifest? That is, what is the salience of a particular identity at any one time? (There are critiques of SIDE that particularly target its abstract notion of anonymity [18]). Second: in what manner do social and personal identities manifest within the agent? We consider only this second obstacle, in the hope that the first is, if not solved, less elusive.

As described in the PECS architecture [19], to adequately represent cognitive mechanisms, perceptions must be filtered, and internal states retrieved. Each agent is prescribed one or more identities. At time-step $t$, the identities filter environmental input and are measured for their salience. The motivations and goals of each identity are then retrieved (representing the internal states of an agent). Via some weighting procedure, the motivation or goal most pertinent to the agent at $t$ is selected as action determining, and the relevant heuristic toolbox then employed (see Fig. 1). (Note that people's differing "self-motion cues" and cognitive maps [e.g. 21, 22], and given the "many-motivations, one goal; many-goals, one motivation' phenomenon [20], means motivation must be addressed separately to goal-pursuance.) It is this weighting procedure, we propose, that could unveil the nuances of group behaviour.

At a finer level, the specific effects of social and personal identity variables are open to investigation. [1] present some of the latest methodologies, in particular, the popular communication heuristic prescribed by [9]. We may also consider the empirical investigation of [23], which suggests an ellipse of minimum area shapes groups. [24] suggest a distinction between in-group cohesion and in-group sociality. Considering cohesion independently allows us to introduce agents' personal (identity) and social (identity) space requirements, e.g. [25]. Whilst [26] set-out a means of in-group imitation by considering Social Comparison Theory: in our context, certain identities are shared or copied amongst in-group members. Note also that by deriving variables that traverse social and personal identities, the salience of a specific identity at any one time will bear no influence on an agent's base-level functioning. In any case, the modular framework prescribed by [6] should include empirically reflective heuristics that manage the wishes of distinct and interactive agent identities.

In attempting to simulate pedestrian behaviour, practitioners have struggled to encompass the widely varying and deeply complex dynamics of social groups. It is hoped this identity-based approach could aid future projects in binding many available methodologies and theories in group dynamics modelling.

This work was funded by the German Federal Ministry of Education and Research through the project S2UCRE.

\section{References}

[1] Cheng L, Yarlagadda R, Fookes C, Yarlagadda P (2014) A review of pedestrian group dynamics and methodologies in modelling pedestrian group behaviours. World Journal of Mechanical Engineering Vol. 1(1), pp. 002-013.

[2] Costa M (2009) Interpersonal Distances in Group Walking. J Nonverbal Behav (2010) 34:15-26

[3] Feng Y, Dewei L (2016) An Emprical Study and Conceptual Model on Heterogeneity of Pedestrian Social Groups for Friend-group and Family-group. Pedestrian and Evacuation Dynamics 2016, Collective Dynamics pp. 402-407 
[4] Zanlungo F, Yücel Z, Brščić D, Kanda T, Hagita N (2017) Intrinsic group behaviour: Dependence of pedestrian dyad dynamics on principal social and personal features. PLoS ONE 12(11): e0187253

[5] Gigerenzer G (2008) Why Heuristics Work. Perspectives on Psychological Science, Vol. 3, No. 1.

[6] Seitz M, Bode N, Köster G (2016) How cognitive heuristics can explain social interactions in spatial movement. Journal of Royal Society Interface, August 2016, vol. 13, Issue 21, DOI:10.1098/rsif.2016.0439

[7] Shah A, Oppenheimer D (2008) Heuristics Made Easy: An Effort-Reduction Framework. American Psychological Association, Psychological Bulletin, 2008, Vol. 134, No. 2, 207-222

[8] Garbarino E, Edell J (1997) Cognitive effort, affect, and choice. J. Consum. Res. 24, 147-158

[9] Moussaïd M, Perozo N, Garnier S, Helbing D and Theraulaz G (2010) The walking behaviour of pedestrian social groups and its impact on crowd dynamics. PLoS ONE 5(4): e10047

[10] Helbing D, Buzna L, Johansson A, Werner T (2005) Self-Organized Pedestrian Crowd Dynamics: Experiments, Simulations, and Design Solutions. Transportation Science 39: 1-24

[11] Moussaïd M, Helbing D, Theraulaz G (2011) How simple rules determine pedestrian behavior and crowd disasters. PNAS April 26, 2011. 108 (17) 6884-6888

[12] Bar M (2007) The proactive brain: Using analogies and associations to generate predictions. Trends in Cognitive Sciences, Volume 11, Issue 9, September 2007, pp. 372

[13] Reicher S, Spears R, Postmes T (1995) A Social Identity Model of Deindividuation Phenomena. European Review of Social Psychology 6(1):161-198

[14] Reicher, S. D. (1984a). The St. Pauls riot: an explication of de limits of crowd action in terms of a social identity model. Europeam Journal of Social Psychology, 14, 1-21

[15] Turner J, Ontaro R (1999) The psychology of the social self: Social identity, personality and the selfconcept: A self-categorization perspective, Chapter 1. Psychology Press, Taylor and Francis Group

[16] Sivers I, Templeton A, Köster G, Drury J, Philippides A (2014) Humans do not always act selfishly: social identity and helping in emergency evacuation simulation. Proceedings of Conference on Pedestrian and Evacuation Dynamics 2014, Transportation Research Procedia 2 585-593

[17] Drury J, Cocking C (2007) The mass psychology of disasters and emergency evacuations: A research report and implications for practice

[18] Moral-Toranzo F, Canto-Ortiz J, Go'mez-Jacinto L (2005) Anonymity effects in computer-mediated communication in the case of minority influence. Computers in Human Behavior 23 (2007) 16601674

[19] Schmidt B (2001) Modelling of Human Behaviour: The PECS Reference Model. AAAI Technical Report FS-01-02

[20] Kielar P, Handel O, Biedermann D, Borrmann A (2014) Concurrent hierarchical finite state machines for modeling pedestrian behavioral tendencies. Transportation Research Procedia 2 ( 2014 ) $576-584$

[21] Golledge R, Jacobson R, Kitchin R, Blades M (2000) Cognitive Maps, Spatial Abilities, and Human Wayfinding. Geographical Review of Japan Vol. 73 (Ser. B), No. 2, 93-104, 2000

[22] Etienne A, Jeffery K (2004) Path Integration in Mammals. HIPPOCAMPUS 14:180 -192

[23] Krüchten C, Schadschneider A (2007) Empirical study on social groups in pedestrian evacuation dynamics. Physica A: Statistical Mechanics and its Applications, vol. 475, pp. 129-141

[24] Jaklin N, Kremyzas A, Geraerts R (2015) Adding Sociality to Virtual pedestrian Groups. Proceedings of Conference: 21 th ACM Symposium on Virtual Reality Software and Technology

[25] Frohnwieser A, Hopf R, Oberzaucher E (2013) Human Walking Behaviour - The Effect of Pedestrian Flow and Personal Space Invasions on Walking Speed and Direction. Human Ethology Bulletin 28 (2013):3 20-28, Brief Reports

[26] Fridman N, Kaminka G (2007) Towards a Cognitive Model of Crowd Behavior Based on Social Comparison Theory. Proceedings of the $22^{\text {nd }}$ national conference on Artificial intelligence - vol. $1 \mathrm{pp}$. 731-737 